\title{
Selective hippocampal lesions disrupt a novel cue effect but fail to eliminate blocking in rabbit eyeblink conditioning
}

\author{
M. TODD ALLEN \\ College of Charleston, Charleston, South Carolina \\ and \\ YAHAIRA PADILLA, CATHERINE E. MYERS, and MARK A. GLUCK \\ Rutgers University, Newark, New Jersey
}

\begin{abstract}
The classical conditioning task of blocking involves the adding of a novel but redundant stimulus to a previously trained stimulus. Both blocking and novelty detection are thought to involve the hippocampus. Previously, Solomon (1977) found that nonselective aspiration lesions of the hippocampal region eliminated blocking in rabbit eyeblink conditioning. We tested the effects of selective ibotenic acid lesions of the hippocampus on blocking, as well as on novelty detection, when training is switched from a tone conditioned stimulus (CS) to a compound tone-light CS in eyeblink conditioning. Selective hippocampal lesions did not eliminate blocking but did lead to a facilitation of conditioned response (CR) acquisition to the tone and to the light, but not to the tone-light compound. Selective hippocampal lesions disrupted a CR decrement observed in sham surgical controls when transferred from tone training to tone-light training. It appears that although selective hippocampal lesions do not eliminate blocking in eyeblink conditioning, they do disrupt novelty detection and may facilitatelearning to a previously blocked cue.
\end{abstract}

The Kamin (1969) blocking effect is one of the most studied classical conditioning tasks. Blocking is a conditioning task in which prior training to one cue (e.g., a tone) reduces learning about a second cue (e.g., a light) in subsequent tone-light compound training, as compared with controls that were trained only to the tone-light compound. Evidence for blocking comes from both reduced conditioned responses (CRs) on test trials of the blocked cue alone and slower learning to the blocked cue during subsequent training, as compared with controls. Blocking involves the addition of a cue that is both novel and redundant, as compared with the cue that had been previously trained.

Various mechanisms have been put forth to account for how the addition of a novel but redundant cue produces the blocking effect. These mechanisms include error correction based on the decreased effectiveness of the unconditioned stimulus (US) to drive learning (Rescorla \&

This work was supported by a grant from the Alzheimer's Association (to M.A.G.), by a Johnson \& Johnson Discovery award (to M.A.G.), by the Office of Naval Research through the Young Investigator Program (to M.A.G.) and Grant N00014-88-K-0112 (to M.A.G.), by a grant from the New Jersey Governor's Council on Autism, and by a National Science Foundation Presidential Early Career Award for Scientists and Engineers (to M.A.G.). Correspondence concerning this article should be addressed to M. T. Allen, Department of Psychology, College of Charleston, Charleston, SC 29424 (e-mail: allenm@ cofc.edu).
Wagner, 1972) and learned inattention to a redundant conditioned stimulus (CS) (Mackintosh, 1973). These mechanisms have since been mapped onto neural circuits known to be involved in various forms of classical conditioning. For example, error correction has been mapped to the cerebellum in the case of motor learning (Gluck, Allen, Myers, \& Thompson, et al., 2001; Kim, Krupa, \& Thompson, 1998) and to the amygdala for fear learning (Fanselow, 1998). CS modulation mechanisms, such as inattention, have been mapped to the hippocampus (Baxter, Gallagher, \& Holland, 1999; Solomon, 1980).

Most empirical studies have supported either an error correction or a learned inattention mechanism. However, a possible interaction between these two mechanisms has been put forth to explain blocking. Holland (1997) proposed that both a hippocampal mechanism of reduction of CS associability and a nonhippocampal mechanism of error correction may be involved in blocking. This theory has been supported by a subsequent empirical study by Baxter et al. (1999) that reported a blocking study in rat appetitive conditioning involving selective cholinergic lesions of the medial septum. These lesions removed the cholinergic innervation to the hippocampus and, thus, disrupted normal hippocampal function. Hippocampal cholinergic disruption did not disrupt blocking, as measured by responding to the blocked cue alone on test trials, but did disrupt blocking when tested by subsequent training of the blocked cue. This finding was interpreted so that the 
hippocampus is involved in reducing the ability of a CS to enter into new associations but is not involved in altering US effectiveness through a process of error correction.

This evidence for multiple mechanisms interacting to produce blocking fits in with the interactions between the cerebellar and the hippocampal systems known to be involved in eyeblink conditioning(Allen, Myers, \& Gluck, 2001). Eyeblink conditioning is an associative learning paradigm in which a neutral stimulus, such as a tone or a light (the CS), is paired with a response-evoking stimulus, such as a corneal air puff (the US). Initially, the CS elicits no behavioral response, whereas the US elicits a protective eyeblink response. By repeatedly pairing the CS followed by the US, the CS alone comes to elicit an anticipatory eyeblink response (the CR). For a complete review of the behavioral eyeblink conditioning literature, see Gormezano, Kehoe, and Marshall (1983).

Blocking has had a long history in eyeblink conditioning (Kim et al., 1998; Kinkaide, 1974; Marchant \& Moore, 1973; Solomon, 1977). Recent work has focused on the error correction mechanism (Gluck et al., 2001; Kim et al., 1998), but earlier blocking experiments in eyeblink conditioning focused on a hippocampal mechanism that involves inattention or inhibition (Solomon, 1977).

In the work presented here, we tested the role of the hippocampus in rabbit eyeblink conditioning. Solomon (1977) reported that aspiration lesions of the dorsal hippocampal region eliminated blocking in rabbit eyeblink conditioning. However, although Solomon (1977) targeted only the dorsal hippocampus, these lesions were nonselective aspiration lesions that also damaged areas of overlying cortices, such as the cingulate, occipital, parietal, prefrontal, and retrosplenial cortices. These aspiration lesions may have damaged hippocampal afferents from the entorhinal cortex as well. Damage to axons from the entorhinal cortex to hippocampal regions CA3 and CA1 may have disrupted normal entorhinal function as well. This unintended damage to surrounding regions of the cortex makes it difficult to conclude that the effects of the hippocampal lesions on blocking in eyeblink conditioning reported by Solomon (1977) were due exclusively to hippocampal damage.

In addition, recent work from our laboratory indicates that selective hippocampal lesions do not have the same effect as nonselective hippocampal region lesions (Solomon \& Moore, 1975), at least for preexposure tasks such as latent inhibition (Shohamy, Allen, \& Gluck, 2000) and learned irrelevance (Allen, Chelius, \& Gluck, 2002). These tasks were hypothesized to involve the same mechanism of learned inattention as blocking (Solomon, 1977). This same pattern of results, in which selective hippocampal lesion effects differ from nonselective hippocampal region lesion effects, may hold true for blocking as well.

Recent computational models have predicted that a selective hippocampal lesion should not disrupt blocking (Myers, Gluck, \& Granger, 1995; Schmajuk \& Buhusi,
1997). This prediction is due to the assumption by both of these models that a neural substrate other than the hippocampus is responsible for blocking through a mechanism of error correction. Myers et al. hypothesized that error correction occurs in the cerebellar system, while Schmajuk and Buhusi hypothesized that error correction occurs in the entorhinal cortex. In either case, blocking in eyeblink conditioning is assumed to involve some error correction mechanism that is independent of the hippocampus.

To examine the role of the hippocampus in blocking, we applied the ibotenic acid selective lesion technique, developed in the rat by Jarrard (1989), to rabbits. Recent work in our laboratory has demonstrated the effectiveness of this selective lesion technique in the rabbit (Allen, Chelius, \& Gluck, 2002; Allen, Padilla, \& Gluck, 2002; Shohamy et al., 2000). This selective lesion technique allowed us to remove the dorsal area of the dorsal hippocampus removed by Solomon (1977), while leaving the overlying cortex and fibers of passage within the hippocampus intact. The implementation of selective hippocampal lesions to test the role of the hippocampus in blocking would allow us to determine whether the hippocampus itself is necessary for blocking in rabbit eyeblink conditioning. We hypothesized that, in contrast to the previous nonselective hippocampal lesion results from Solomon (1977), a selective lesion of the dorsal hippocampus would not disrupt blocking in delay eyeblink conditioning in the rabbit.

We also wanted to test two other hypotheses regarding blocking that involve the effects of the addition of a novel cue on expression of a previously learned CR. If an animal is trained to a tone and then switched to compound tone-light training, the light is a novel cue but is also redundant to the tone as a signal for the US. The Rescorla-Wagner (1972) rule predicts that there will be no novelty effect in response to the added cue during blocking. Since Rescorla and Wagner hypothesized that learning is based on error correction owing to unpredicted USs, if the US is predicted by the previously trained cue, there is no error to drive learning to any added cue that is redundant to the previously trained cue. Therefore, if blocking in eyeblink conditioning is due to a purely error correction mechanism, there will be no difference in CRs when a novel cue is added to a previously trained cue.

Conversely, Mackintosh and Turner (1971) predicted that initially, the added cue is attended to owing to its novelty but that it is then quickly inattended to, owing to its redundancy with the previously trained cue. Therefore, if blocking involves some form of CS modulation, there should be a decrement in CRs when a novel cue is added to a previously trained cue. We hypothesized, on the basis of Baxter et al.'s (1999) result, that blocking in eyeblink conditioning could involve some form of CS modulation that is independent of error correction in the cerebellar system and would exhibit a decrement in CRs from the addition of a novel cue. 
Table 1

Stereotaxic Coordinates for

Selective Dorsal Hippocampal Lesions

\begin{tabular}{ccc}
\hline A-P & M-L & D-V \\
\hline-4.0 & \pm 3.0 & $-5.2,-3.2$ \\
& \pm 5.0 & $-5.2^{*},-3.2$ \\
-5.0 & \pm 4.0 & $-5.2,-3.2^{*}$ \\
& \pm 7.5 & $-5.7^{*},-3.5$ \\
-6.0 & \pm 4.5 & $-5.5,-3.8^{*}$ \\
& \pm 8.0 & $-6.5^{*},-4.2$ \\
\hline
\end{tabular}

Note-Bregma is used as the zero point for the A-P and M-L coordinates, whereas the $\mathrm{D}-\mathrm{V}$ measures are taken from dura. Bregma is $1.5 \mathrm{~mm}$ ventral to lambda. The volume of injection for the hippocampal lesion is 0.15 or $0.10\left(^{*}\right) \mu \mathrm{L}$ at each site.

The second novel cue hypothesis is that if there is a novelty effect during blocking, it should be hippocampal dependent. Several studies have supported the role of the hippocampus in detecting and encoding representations of novel stimuli (Grossberg \& Merrill, 1992; Menon, White, Eliez, Glover, \& Reiss, 2000; Miller \& Matzel, 1988; Pribram, 1986; Squire, 1992). Eichenbaum (1999) defined novelty detection as a complex process that requires some process of matching or failing to match a stimulus to a stored representation. The detection of novelty probably involves some procession to encode the relationships among the elements that compose the novel stimulus. This matching of stimuli to stored representations and the subsequent encoding of complex stimulus fits with the role of the hippocampus in classical conditioning as hypothesized in models and theories of the hippocampus (Bunsey \& Eichenbaum, 1993; Gluck \& Myers, 1993; Vinogradova, 2001). An alternate theory is that novelty detection does not take place in the hippocampus but takes place in the nucleus accumbens (Buhusi \& Schmajuk, 1996).

If the hippocampus is involved in the detection of novel stimuli on the basis of a comparison with representations of previously learned stimuli, the selective removal of the hippocampus should disrupt this comparison, and there should be no effect of the novel cue of a previously learned CR. We hypothesized that an initial decrement in conditioned responding that would occur in normal rabbits when switched from tone training to tone-light training would not occur in rabbits with selective hippocampal lesions. Overall, we predicted that rabbits with selective hippocampal lesions would exhibit normal blocking but would not exhibit any decrement in CRs in response to the addition of the novel cue.

\section{METHOD}

\section{Subjects}

Twenty-four male rabbits were used in this experiment. The rabbits were purchased from Covance Laboratories (Princeton, NJ). The rabbits were housed in individual cages in the AAALAC-approved Rutgers University Animal Facility, Newark, NJ. They were given free access to food and water. They were maintained on a 12:12-h light:dark cycle, with lights on at 7:00 a.m. All testing occurred during the light cycle. All procedures and protocols were approved by the Rutgers IACAUC (Protocol 96-010).

\section{Surgery}

All the rabbits underwent aseptic surgery for either a selective dorsal hippocampal lesion by injection of ibotenic acid or a sham lesion by injection of the vehicle solution alone. The rabbits were anesthetized via a subcutaneous (s.c.) injection of xylazine $(6 \mathrm{mg} / \mathrm{kg}$ ) followed, $15 \mathrm{~min}$ later, by an intramuscular (i.m.) injection of ketamine $(60 \mathrm{mg} / \mathrm{kg})$. The rabbit was then placed in a stereotaxic head holder. Additional i.m. $1 \mathrm{cc}$ doses of a ketaminexylazine (2:1) mixture were administered at $1-\mathrm{h}$ intervals.

During the surgery, two holes were drilled bilaterally into the skull 3-8 mm lateral and 3-6 mm posterior of bregma. Selective hippocampal lesions were made by injecting ibotenic acid $(10 \mu \mathrm{g} / \mu \mathrm{L})$ into 24 injection sites bilaterally into the dorsal hippocampus with an injection of 0.15 or $0.10 \mu \mathrm{L}$ of solution per injection site. Stereotaxic coordinates and specific injection sizes are listed in Table 1.

Following the injections, the holes in the skull were sealed with bone wax. Two stainless steel screws were mounted in the skull to anchor the headstage. The exposed skull was covered with dental acrylic to form the headstage. A bolt was attached in the acrylic for the mounting of the eyeblink detector assembly during conditioning. The rabbits were allowed a week for recovery from the surgery prior to the initiation of behavioral conditioning.

\section{Materials}

For training, each rabbit was restrained in Plexiglas restraint boxes in individual conditioning chambers. Each chamber contained a speaker, an air hose assembly, and an eyeblink detector assembly (L.T. Thompson, Moyer, Akase, \& Disterhoft, 1994). The stimuli and recording of the behavior were computer controlled (for technical details, see Chen \& Steinmetz, 1998).

\section{Behavioral Response Assessment}

Eyeblinks were monitored with an opto-electronic sensor that consisted of a light-emitting diode (LED) and a phototransistor (for technical details, see L.T. Thompson et al., 1994). The LED emitted a beam of infrared light that was reflected off the cornea, and the reflectance of this beam was converted to a DC voltage by a phototransistor. The eyeblink signal was filtered (between $0.1 \mathrm{~Hz}$ and $1 \mathrm{KHz})$ and amplified $(100 \times)$ by a differential AC amplifier (A-M Systems, Everett, WA).

When the rabbit closed its eye, the reflectance of the infrared beam changed and was recorded as an eyeblink. Any movement greater than $0.5 \mathrm{~mm}$ during the pre-CS period caused the training trial to be discarded from analysis. A CR was scored if movement greater than $0.5 \mathrm{~mm}$ was seen in the CS period. Each trial's behavioral record was displayed on the computer screen. The computer analyzed the behavioral data and delivered the data for each block of 50 trials.

\section{Stimuli}

The CS was either a 450-msec, 90-dB, 1000-Hz tone or a 450-msec, 12-V light. The US was a 50-msec, 4-psi corneal air puff delivered via a rubber hose attached to the eyeblink detector assembly and aimed at the rabbit's cornea. The CS and US coterminated so that there was an interstimulus interval (ISI) of $400 \mathrm{msec}$. The intertrial interval (ITI) was a pseudorandom interval that averaged $30 \mathrm{sec}$ and ranged from 25 to $35 \mathrm{sec}$. All training was done in the darkened chambers, with a red light outside in the conditioning room.

\section{Design and Procedure}

Adaptation. Prior to acquisition, the rabbits were adapted to the conditioning chamber and restraint box for two daily sessions for $30 \mathrm{~min}$ on the first session and $45 \mathrm{~min}$ on the second session.

Acquisition. Acquisition consisted of four phases. Hippocampallesioned and sham-lesioned rabbits were divided equally between a blocking and a control condition.

Phase 1 consisted of 700 trials over seven consecutive daily sessions. The rabbits in the blocking condition received tone-air-puff 
training. The rabbits in the control condition received exposure to the conditioning context, but with no stimuli presented for seven consecutive daily sessions. Phase 2 consisted of 500 trials over five consecutive daily sessions. All the rabbits received tone-light-airpuff training, with the tone and the light presented simultaneously and with an ISI of $400 \mathrm{msec}$ between the onset of the tone-light and the onset of the air puff. Phase 3 consisted of 30 light-alone test trials given to all the rabbits. Phase 4 consisted of 500 trials of light and air puff training given to all the rabbits over five consecutive daily sessions

Histology . Following completion of conditioning, all the rabbits were overdosed by an IV injection of pentobarbital into the marginal ear vein and were perfused via the ascending aorta with a $0.9 \%$ saline solution followed by a $10 \%$ formalin solution. The brain was then removed and preserved in a $30 \%$ sucrose/ $10 \%$ formalin solution. The brain was sliced into 80-micron sections on a freezing microtome, mounted on slides, and stained with the cresyl violet method.

\section{Data Analysis}

Behavioral results in the form of percentage of CRs (\%CR) for all phases were analyzed with a repeated measures analysis of variance in 50 trial blocks, with the exception of the data from Phase 3, which were analyzed as a single block of 30 trials. We assessed the size and location of the hippocampal lesions by viewing the slice under a microscope and drawing the lesion onto a template of a corresponding sham-lesioned hippocampus. The area of the lesion was then calculated as a percentage of the area of the sham-lesioned hippocampus.

\section{RESULTS}

\section{Histology}

As was previously discussed, the hippocampal lesions in the blocking study of Solomon (1977) were nonselective aspiration lesions that targeted the dorsal hippocampus. In Figure 1, we compare the location and extent of the minimal (black) and maximal (vertical lines) lesions for our selective dorsal hippocampal lesions with the histology presented by Solomon (1977) for his hippocampal lesion blocking study. As can be observed in Figure 1, the location and extent of our selective lesions match with those of Solomon (1977). In both cases, the majority of damage is to the dorsal hippocampus, with some damage to the more caudal and ventral portions of the hippocampus, but with no damage to the ventral hippocampus in the rostral portion.

Overall, the mean area of the selective dorsal hippocampal lesions were determined to be $74 \%(S E=5.8 \%)$ of the normal area of a sham-lesioned hippocampus for the left side and $72 \%$ ( $S E=4.5 \%$ ) for the right side. Representative photomicrographs of a sham-lesioned dorsal hippocampus and a selectively lesioned dorsal hippocampus are shown in Figures 2A and 2B, respectively. The ibotenic acid lesion technique, along with the specific coordinates we used, resulted in a dorsal hippocampal lesion that was very complete and yet spared the overlying cortex. The selectively lesioned hippocampal and sham-lesioned hippocampus with the overlying cortex intact in both cases are shown in Figure 2.

In Figure 3 we show a set of magnifications $(100 \times)$ of a selective dorsal hippocampal lesion, as compared with a sham-lesioned control, to demonstrate the extent of loss of neurons within CA1, CA3, and the dentate gyrus in the dorsal hippocampus. Therefore, through our selective ibotenic acid lesions of the dorsal hippocampus, we have obtained a lesion that did not damage the surrounding cortical regions and resulted in a complete loss of neurons in the dorsal hippocampus.

\section{Behavioral Results}

All the rabbits were able to learn to exhibit CRs to the tone, the light, and the tone-light compound. The learning curves for Phase 1 are shown in Figure 4A. There was a significant difference in conditioned responding across sessions $[F(13,130)=59.636, p<.001]$, indicating that all the rabbits exhibited more CRs to the tone as training in Phase 1 progressed. There were significantly more CRs exhibited by the rabbits in the selective hippocampal-lesioned group than by those in the shamlesioned group $[F(1,10)=6.22, p<.05]$. There was a group $\times$ lesion interaction $[F(13,130)=2.436, p<.01]$ so that the two lesion groups differed early in training, but by the end of Phase 1, all the rabbits were exhibiting asymptotic levels of CRs (over $80 \%$ ) to the tone.

The learning curves for Phase 2 are shown in Figure $4 \mathrm{~B}$. There were significant differences in conditioned responding across sessions $[F(9,180)=31.593$, $p<.001]$, so that all the rabbits exhibited more CRs to the tone-light compound as training in Phase 2 progressed. There were significantly more CRs exhibited by rabbits in the blocking condition than by those in the control condition $[F(1,20)=13.481, p<.005]$. There were no significant differences in conditioned responding between the hippocampal-lesioned rabbits and the shamlesioned rabbits $[F(1,20)=2.6696, p>.11]$. There were no significant interactions in Phase 2, $p>05$.

The \%CRs to the light test trials for Phase 3 are shown in Figure 4C. Overall, there were significantly more CRs exhibited by the rabbits in the blocking and control conditions $[F(1,20)=8.539, p>.01]$. There were no significant differences in conditioned responding to the light between the hippocampal-lesioned rabbits and the sham-lesioned rabbits $[F(1,20)=0.089, p<.75]$. There was also no interaction between the blocking and control conditions and the lesion groups $[F(1,20)=0.2, p<.65]$.

The learning curves for Phase 4 are shown in Figure 4D. There was a significant difference in conditioned responding across sessions $[F(9,180)=11.439, p<$ $.001]$, so that all the rabbits learned to perform CRs to the light as Phase 4 progressed. There were significantly more CRs exhibited by the rabbits in the control condition than by the rabbits in the blocking condition $[F(1,20)=$ $14.083, p<.001]$. There were no significant differences in CRs to the light test trials between the hippocampallesioned rabbits and the sham-lesioned rabbits $[F(1,20)=$ $3.658, p \geq .07]$. There was a session $\times$ group interaction $[F(9,180)=1.963, p<.05]$, so that early in Phase 4 , there were significant differences between the rabbits in the blocking condition and the rabbits in the control con- 
A
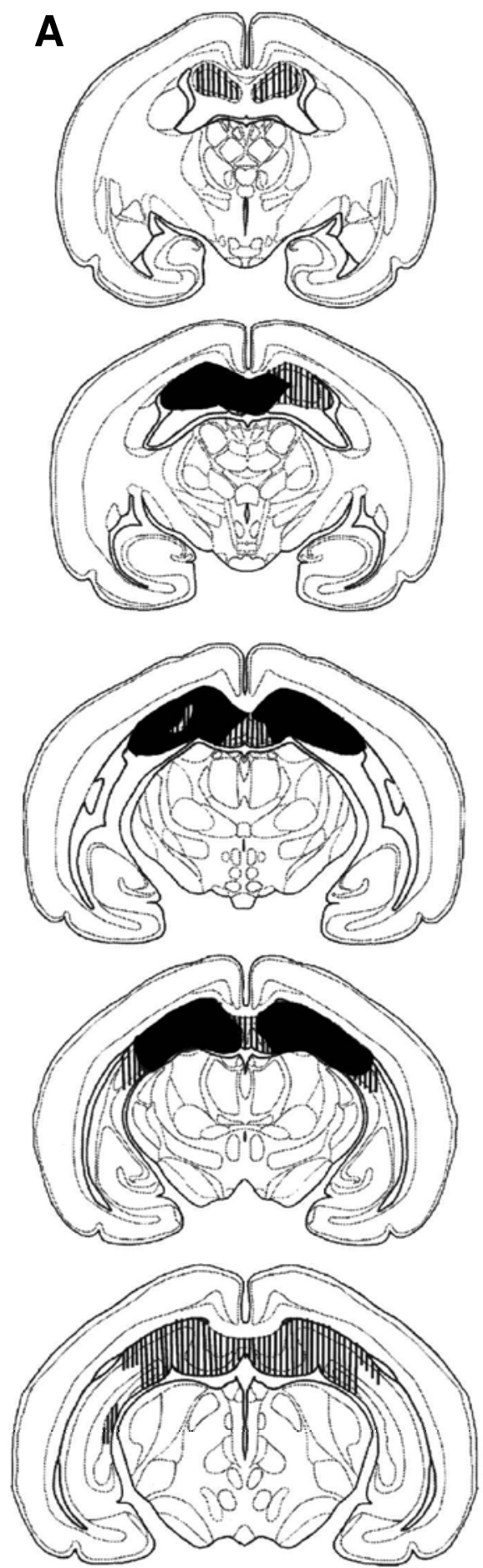

B
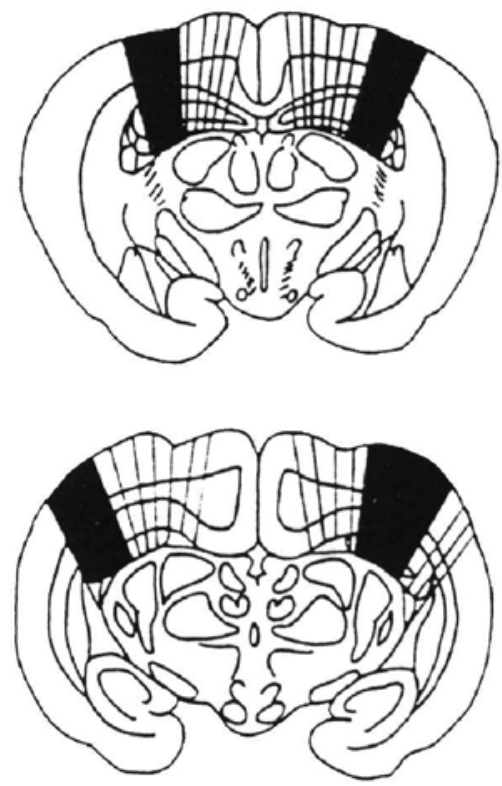

Figure 1. The extent and location of the minimal (black) and the maximal (vertical lines) lesions for our selective dorsal hippocampal lesions and for comparable lesions from Solomon (1977). The sections are arranged in a rostral-caudal orientation as one progresses down each column. (A) The selective dorsal hippocampal lesions produced by injections of ibotenic acid for the present study. Overall, our selective dorsal hippocampal lesions damaged areas of the hippocampal directly comparable to those of Solomon (1977). Our selective dorsal hippocampal lesions also extended further in the rostralcaudal plane than did those of Solomon (1977). (B) The nonselective dorsal hippocampal lesions produced by aspiration from Solomon's (1977) study of blocking. 
A

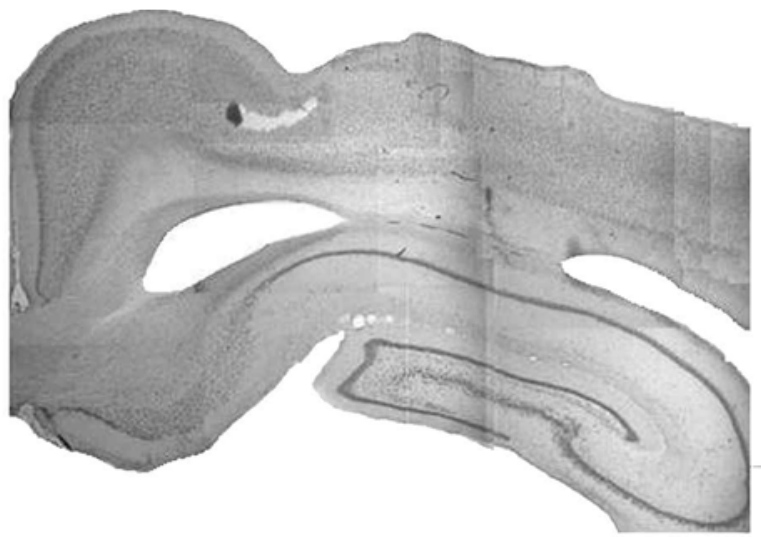

B

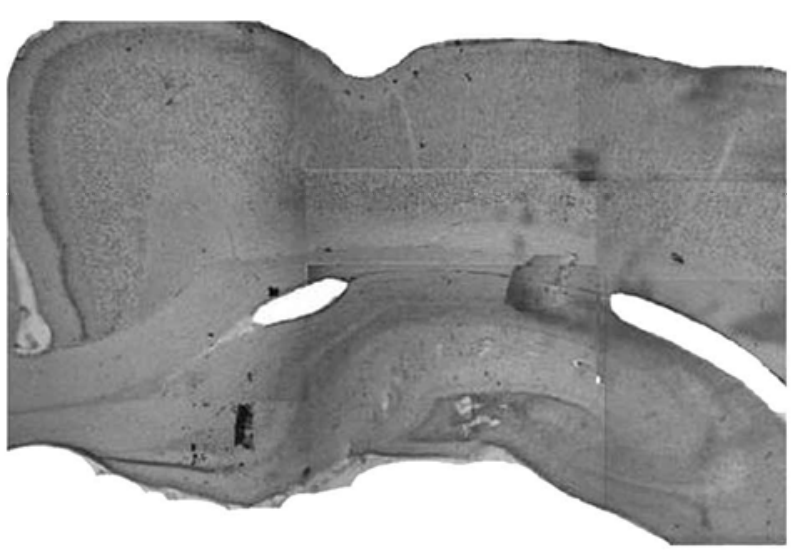

Figure 2. The results from the histology of the selective hippocampal and sham lesions. In both cases, the overlying cortex is intact. (A) A photomicrograph of a sham-lesioned dorsal hippocampus in which only the vehicle solution was injected. (B) A photomicrograph of a selectively lesioned dorsal hippocampus made by injections of ibotenic acid $(0.15$ or $0.10 \mu \mathrm{L}$ of a $10 \mu \mathrm{g} / \mu \mathrm{L}$ solution).

dition, but these differences disappeared by the middle of training to the light. There were no other significant interactions in Phase 4.

However, if only the hippocampal-lesioned and the sham-lesioned rabbits in the blocking conditions were compared, as was the case in Phase 1, there were significantly more CRs exhibited by hippocampal-lesioned rabbits than by the sham-lesioned rabbits $[F(1,10)=11.937$, $p<.01]$.

\section{Novel Cue Effects}

We tested the hypotheses concerning a CR decrement by comparing \%CR for the last 10 trials of tone training in Phase 1 and for the first 10 trials of tone-light training in Phase 2 for both the hippocampal blocking group and the sham blocking group, as is shown in Figure 5. Overall, there were no significant differences between conditioned responding in Phase 1 and Phase $2[F(1,10)=$
4.034, $p=.072]$. There were no significant differences between the hippocampal-lesioned and the sham-lesioned groups $[F(1,10)=0.937, p>.35]$. However, there was a significant interaction of phase and group $[F(1,10)=$ $10.793, p<.01]$. This interaction was furthered analyzed by paired and individual $t$ tests. There was a significant decrease in CRs between the end of Phase 1 and the beginning of Phase 2 for the sham blocking group [paired $t(5)=-3.542, p<.05$ ], but not for the hippocampal lesion blocking group [paired $t(5)=-0.961, p>$ .38]. The percentage of CRs between the sham and the hippocampal blocking groups for the last block of Phase 1 and the first block of Phase 2 was compared with an independent measure $t$ test. There was a significant difference between the sham and the hippocampal lesion groups for the first block of Phase 2 [individual $t(5)=$ $-2.659, p<.05]$, but not for the last block of Phase 1 [individual $t(5)=1.290, p>.25$ ]

\section{DISCUSSION}

Overall, the major finding of this experiment was that selective ibotenic acid lesions of the dorsal hippocampus did not eliminate blocking. This is in contrast to the finding of Solomon (1977) that nonselective aspiration lesions of the dorsal hippocampus disrupted blocking.

Our purpose in this experiment was to determine whether lesioning of the hippocampus itself was necessary for blocking in rabbit eyeblink conditioning by the use of ibotenic acid. Analysis of the present lesions indicates that we have achieved the desired damage. Specifically, we selectively lesioned the same area of the hippocampus as Solomon (1977) had previously lesioned nonselectively. We selectively removed the CA1, CA3, and the dentate gyrus regions in the dorsal hippocampus, while leaving the overlying cortex intact.

We will now discuss the specific results for each phase in order. In Phase 1, all the selective hippocampal-lesioned rabbits and sham-lesioned rabbits learned to exhibit CRs to the tone. The hippocampal-lesioned rabbits exhibited accelerated learning to the tone, as compared with the sham-lesioned rabbits. This was not an unexpected finding. Previously, Schmaltz and Theios (1972) found that hippocampal-lesionedrabbits exhibited accelerated learning to a tone in eyeblink conditioning. This acceleration following a hippocampal lesion has been explained as the effect of releasing the cerebellum from hippocampal inhibition. There is a long history of theories going back over 30 years that suggest a specialized role for the hippocampus in tasks that involve inhibition or learned inattention (Chan, Morell, Jarrard, Davidson, 2001; Douglas, 1967; Douglas \& Pribram, 1966; Kimble, 1968; Solomon, 1980).

The role of the hippocampus in inhibition can be understood on the basis of the type of conditioning tasks that require hippocampal processing. Overall, the simple CS-US associations of delay conditioning can be done by the cerebellar circuitry delineated by R. F. 
A

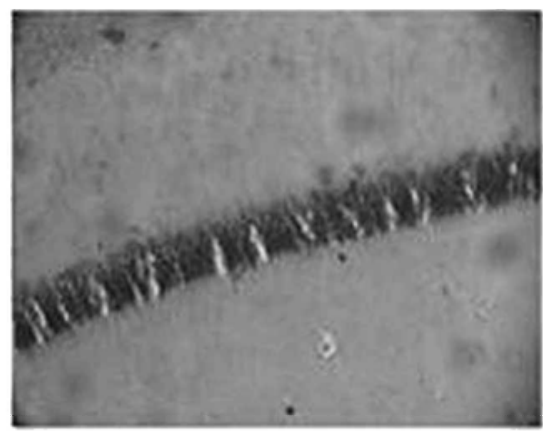

C

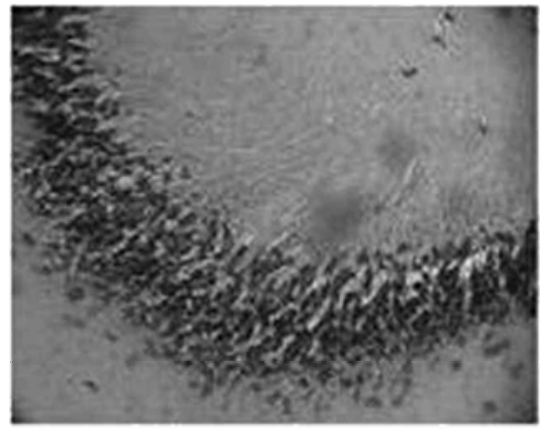

E

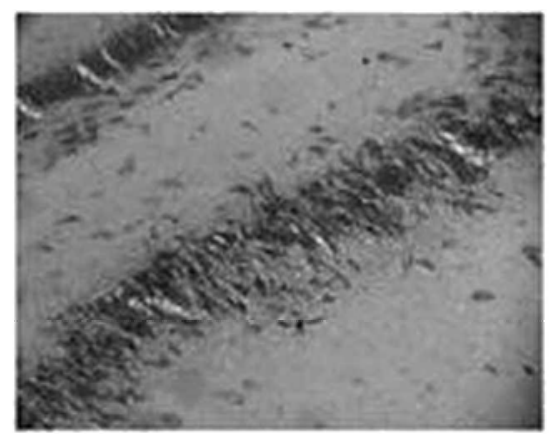

B

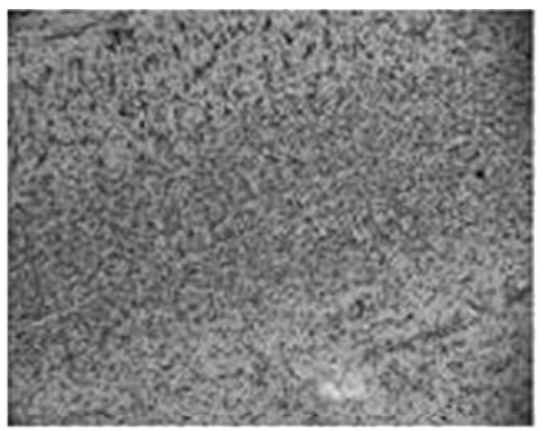

D

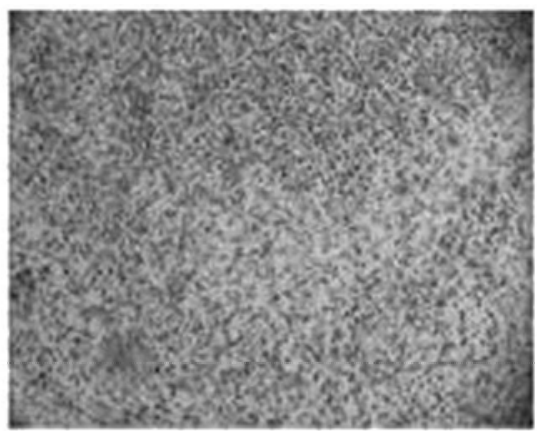

$\mathbf{F}$

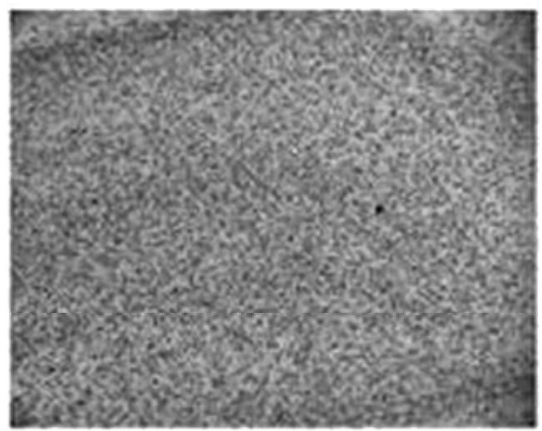

Figure 3. Extent of neuronal loss in the CA1 and CA3, and dentate gyrus regions in the dorsal hippocampus from ibotenic acid lesions. All photomicrographs are at a magnification of $200 \times$. (A) A photomicrograph of the CA1 region of a sham-lesioned hippocampus, showing the strongly stained pyramidal cell layer. (B) A photomicrograph of the CA1 region of a selectively lesioned hippocampus. Note the absence of pyramidal cells, as compared with panel A. (C) A photomicrograph of the CA3 region of a sham-lesioned hippocampus, showing the strongly stained pyramidal cell layer. (D) A photomicrograph of the $\mathrm{CA} 3$ region of a selectively lesioned hippocampus. Note the absence of neuronal staining, as compared with panel C. (E) A photomicrograph of the dentate gyrus region of a shamlesioned hippocampus, showing the strongly stained neurons. (F) A photomicrograph of the dentate gyrus region of a selectively lesioned hippocampus. Note the absence of neuronal staining, as compared with panel $\mathbf{E}$.

Thompson (1986). The hippocampus is necessary for more complex tasks that require adaptable representations of stimuli and their relationships. Therefore, for the hippocampus to form these relationships, it inhibits the formation of the simple CS-US association by the cerebellum in order for the hippocampus to be able to transmit the complex representations to the cerebellum, to be used in the formation of the proper CS-US association.

The Gluck and Myers (1993) model accounts for a hippocampal lesion's facilitating formation of a simple
CS-US through a different interpretation. With an intact hippocampus, there is learning of a CS-US association from representations that are changing on the basis of hippocampal alterations of stimulus representations. In the case of a hippocampal lesion, the stimulus representations are fixed throughout learning. We concluded that learning with such a fixed stimulus representation should be faster and more complete than trying to learn with a randomly changing representation (Gluck \& Myers, 1993). 
A
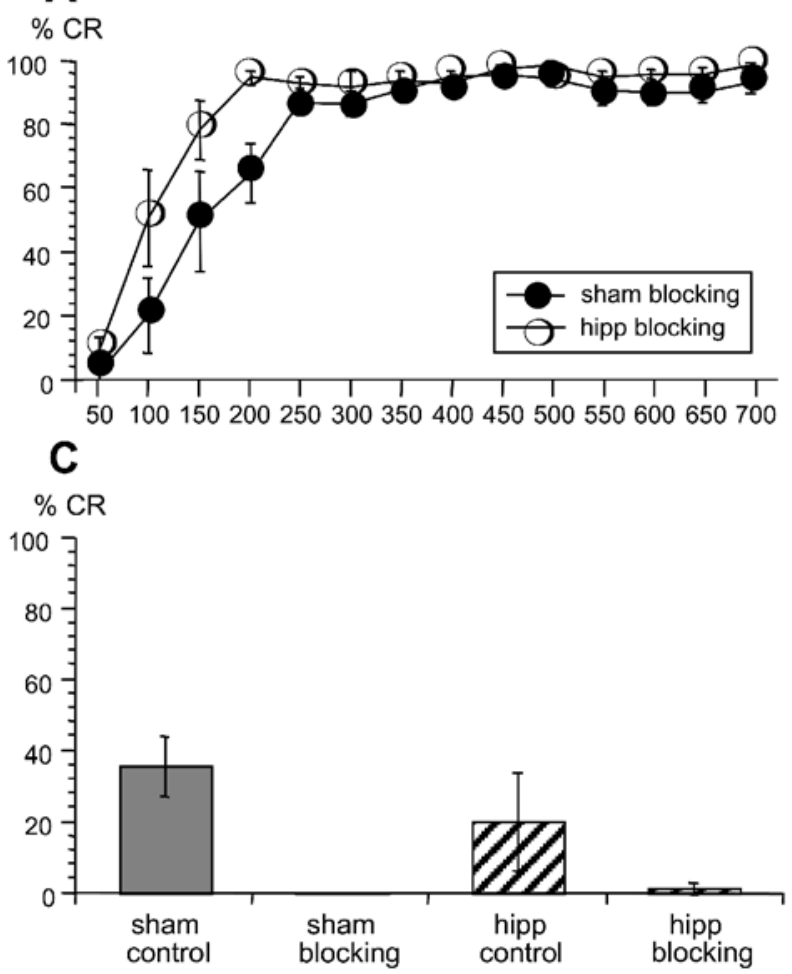

B
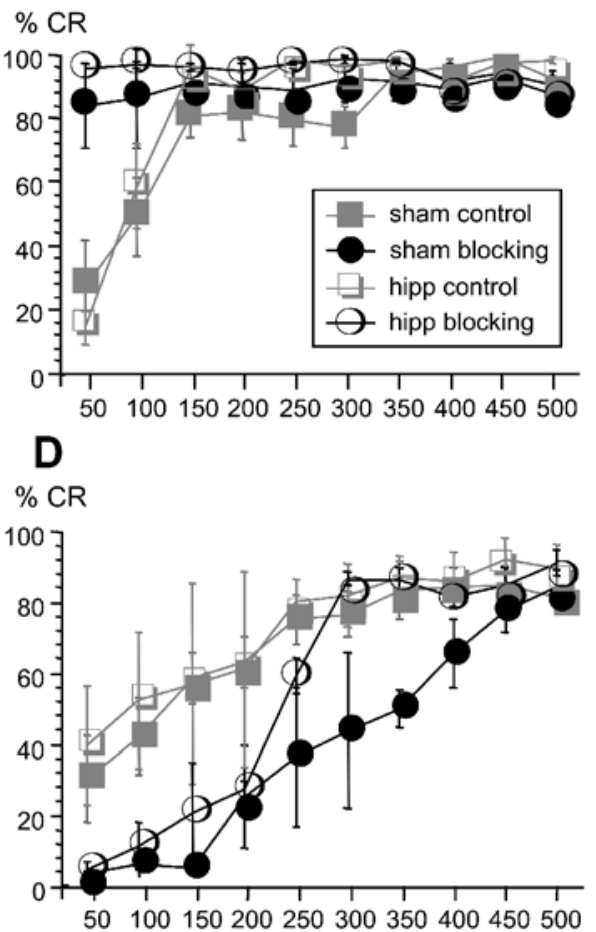

Figure 4. The behavioral results for all four phases of blocking. (A) The behavioral results for Phase 1 (tone-airpuff training). Overall, both the sham-and the hippocampal-lesioned rabbits in the blocking condition learned with the tone. The sham- and the hippocampal-lesioned rabbits in the control condition received no stimuli in Phase 1. (B) The behavioral results for Phase 2 (tone-light-air-puff training). Overall, the sham- and the hippocampallesioned rabbits in the blocking condition continued to exhibit conditioned responses to the tone-light compound across all of Phase 2. The sham- and the hippocampal-lesioned rabbits in the control group learned to give conditioned responses to the tone-light compound. (C) The behavioral results for Phase 3 (light-alone test trials). Overall, the sham- and the hippocampal-lesioned rabbits exhibited blocking, in that there was more responding to the light in the rabbits trained in the control condition than in the rabbits trained in the blocking condition. (D) The behavioral results for Phase 4 (light-air-puff training). Overall, the sham- and the hippocampal-lesioned rabbits in the control condition learned with the light faster than both the sham-and the hippocampal-lesioned rabbits in the blocking conditioning. However, by the end of Phase 4, all the rabbits were exhibiting conditioned responses to the light.

Therefore, removal of the hippocampus, in the case of a simple CS-US association, would result in allowing the cerebellum to be facilitated in acquiring the CR. This idea fits with our findings on the accelerated learning to the tone by the hippocampal lesion group in Phase 1 .

We now turn to the results from the Phase 2 tone-light training. In Phase 2, all the rabbits learned to exhibit CRs to the tone-light compound. Overall, the rabbits in the blocking condition, which had previously received toneair-puff training, continued to respond at asymptotic levels (over $80 \%$ CRs) to the tone-light compound. The rabbits in the control condition, which had not received tone-air-puff training in Phase 1, acquired CRs to the tone-light compound at a normal rate. By the end of Phase 2, all the rabbits were responding to the tone-light compound at asymptotic levels (over $80 \% \mathrm{CRs}$ ). There were no selective hippocampal lesion effects observed in Phase 2. Specifically, there was no facilitation of learning to the tone-light compound in selectively hippocampallesioned rabbits in the control condition.

In Phase 3, both the selective hippocampal-lesioned and the sham-lesioned rabbits in the control condition exhibited more CRs to the light than did the rabbits in the blocking condition. Blocking was observed for both the sham-lesioned and the hippocampal-lesioned rabbits. Selective lesions of the dorsal hippocampus did not disrupt blocking in eyeblink conditioning, as predicted by the computational models of Myers et al. (1995) and Schmajuk and Buhusi (1997).

In Phase 4, both the hippocampal-lesioned and the sham-lesioned rabbits in the control condition exhibited more CRs to the light than did the rabbits in the blocking condition. This is another indication of blocking. There was also a significant difference between the hippocampal-lesionedand the sham-lesioned rabbits in the blocking condition, so that the hippocampal-lesioned rab- 


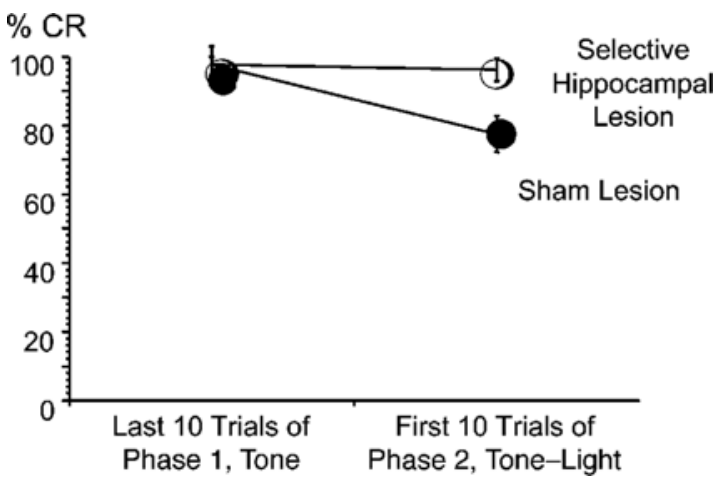

Figure 5. Conditioned response (CR) decrement observed between tone training in Phase 1 and tone-light training in Phase 2. The sham-lesioned rabbits showed a decrement in conditioned responding initially to tone-light presentations following prior tone training. This effect was not observed in the rabbits with selective hippocampal lesions.

bits learned faster to the light than did the sham-lesioned rabbits.

This facilitation of learning to the light in the blocking group could be interpreted as a reduction in the blocking effect that is similar to the results of Baxter et al. (1999), in which hippocampal function was disrupted by the removal of cholinergic inputs to the hippocampus. They found that loss of cholinergic inputs to the hippocampus did not disrupt blocking as measured by light-alone test trials. However, this disruption altered learning to the blocked cue, so that these lesioned animals were facilitated, as compared with normal controls.

However, prior work in our laboratory on the learned inattention mechanism of blocking in rabbit eyeblink conditioning did not provide any evidence that learned inattention was the mechanism for blocking in eyeblink conditioning (Allen, Padilla, \& Gluck, 2002). Normal rabbits that had been previously blocked to a light learned with the light at the same rate as naive controls. Therefore, it is unclear whether blocking in eyeblink conditioning involves both a hippocampal learned inattention mechanism and a nonhippocampal error correction mechanism, as does the appetitive task reported by Baxter et al. (1999).

Although our finding that hippocampal-lesioned rabbits exhibited facilitated learning to the previously blocked cue is consistent with Baxter et al. (1999), it could also be due to a continuation of the hippocampal lesion facilitation effect seen in Phase 1 training to the tone. Therefore, in both phases in which there was a single cue paired with the air puff, hippocampal lesions were facilitated. This overall trend in the data for selective hippocampal lesions to facilitate single CS conditioning tends to lessen the likelihood that the facilitation observed in Phase 4 was due to a reduction in the blocking to the light. There is one difference between the facilitation of training to the tone and training to the blocked light. The facilitation of tone training is evident early in training, whereas the facilitation of training to the blocked light occurs later in training. It still may be that the selective dorsal hippocampal lesions did lessen the blocking effect and that this resulted in faster acquisition of CRs to the blocked cue. Overall, by the end of Phase 4, all the rabbits were exhibiting CRs to the light.

\section{Novel Cue Effects}

A CR decrement was observed during the first 10 trials of tone-light training in the sham-lesioned blocking rabbits that had been switched from tone training. This finding is consistent with the theory of Mackintosh and Turner (1971) that attention is directed to the novel cue in blocking when compound training is initiated. This finding is contrary to the prediction of the RescorlaWagner (1972) rule that predicts that there is no learning to the novel cue, owing to its redundancy to the previously trained cue. Our finding supports the theory that blocking, at least in the case of eyeblink conditioning, is not due purely to an error correction mechanism.

In addition, rabbits with selective hippocampal lesions did exhibit this CR decrement when tone training was switched to tone-light training. This finding fits with theories that propose that the hippocampus is involved in the detection of novel cues during training (Gluck \& Myers, 1993; Vinogradova, 2001). This finding does not support the predictions of others (e.g., Buhusi \& Schmajuk, 1996) who have proposed that novelty was not a hippocampal-dependent task but was dependent on the nucleus accumbens.

Novelty detection within the hippocampus has been theorized to occur through a variety of mechanisms. Hasselmo and Wyble (1997) suggested that the CA3 region of the hippocampus reconstructs inputs from the entorhinal cortex and that the CA1 region of the hippocampus compares the inputs from the entorhinal cortex with the output of CA3. When stimuli are well encoded, there will be a good match between entorhinal cortex inputs and CA3-reconstructed outputs, and hippocampal learning will not be altered. When stimuli are novel and encoding is poor, there is a mismatch between entorhinal cortex inputs and CA3-reconstructed outputs, and hippocampal learning will be increased to correct this error in stimulus encoding. Vinogradova (2001) suggested that the CA3 region of the hippocampus detects novelty by comparing signals from the subcortex via the fornix and those from the cortex via the entorhinal cortex. No matter through which specific mechanism, it appears that the hippocampus is involved in the detection of novel cues during the blocking task. Future work should determine exactly what mechanisms within the hippocampus are involved in the detection of stimulus novelty.

The results from this paper support the idea that blocking involves some hippocampal function independent of error correction within the cerebellar system. Blocking in selectively hippocampal-lesioned rabbits is not identical to blocking in sham-operated controls. Blocking was intact, as was indicated by test trials of the blocked light. However, subsequent training to the blocked cue in selectively hippocampal-lesioned rabbits was facilitated. 
This pattern of results fits with prior findings by Baxter et al. (1999). In addition, these findings support the predictions from computational models (Myers et al., 1995; Schmajuk \& Buhusi, 1997) that a selective hippocampal lesion would not eliminate blocking in rabbit eyeblink conditioning. The fact that our selective hippocampal lesions did not disrupt blocking, whereas prior nonselective hippocampal lesions disrupted blocking (Solomon, 1977), may be due to the role of nonhippocampal structures within the hippocampal region, such as the entorhinal cortex. Schmajuk (Schmajuk \& Buhusi, 1997) proposed that the entorhinal cortex is necessary for blocking. Prior work in this laboratory has indicated that selective entorhinal lesions have the same disruptive effect as hippocampal region lesions on such tasks as latent inhibition (Shohamy et al., 2000) and learned irrelevance (Allen, Chelius, \& Gluck, 2002). Future work should test these predictions, to determine whether indirect damage to the entorhinal cortex is responsible for Solomon's (1977) finding of hippocampal region lesions' disrupting blocking.

One may argue that the failure of our selective dorsal hippocampal lesions to disrupt blocking was due simply to not lesioning the entire hippocampus. As was previously mentioned, the hippocampal region lesions that Solomon (1977) found disrupted blocking damaged the dorsal hippocampus, but not the ventral hippocampus. Therefore, the difference between our failure to find that selective hippocampal lesions disrupted blocking and Solomon's (1977) finding that nonselective hippocampal lesions disrupted blocking cannot be attributed to the dorsal nature of the lesions. It may be that Solomon's (1977) lesions, owing to their nonselective nature, did indirectly damage some ventral hippocampal or other brain region; this other possible damage was not addressed by the histology of Solomon (1977).

We can conclude that the selective dorsal hippocampal lesions were effective in producing two expected hippocampal lesion effects: a facilitation of single CS conditioning (Schmaltz \& Theios, 1972) and a disruption of a $\mathrm{CR}$ decrement in response to the addition of a novel cue to a previously trained cue. The failure of these lesions to disrupt blocking is probably due to lack of damage to cortical structures that were damaged by Solomon's (1977) nonselective aspiration lesions, possibly the entorhinal cortex. Future work should examine more precisely the role of the entorhinal cortex in blocking, in order to expand our understanding of the neural substrates of blocking in eyeblink conditioning.

\section{REFERENCES}

Allen, M. T., Chelius, L., \& Gluck, M. A. (2002). Selective entorhinal lesions and nonselective cortical-hippocampal region lesions, but not selective hippocampal lesions, disrupt learned irrelevance in rabbit eyeblink conditioning. Cognitive, Affective, \& Behavioral Neuroscience, 2, 214-226.

Allen, M. T., Myers, C. E., \& Gluck, M. A. (2001). Parallel neural systems for stimulus selection in classical conditioning: Support from computational modeling. Integrative Physiological \& Behav- ioral Science, 36, 36-61.

Allen, M. T., Padilla, Y., \& Gluck, M. A. (2002). Ibotenic acid lesions of the medial septum retard delay eyeblink conditioning in rabbits. Behavioral Neuroscience, 116, 733-738.

Baxter M. G., Gallagher M., \& Holland P. C. (1999). Blocking can occur without losses in attention in rats with selective removal of hippocampal cholinergic input. Behavioral Neuroscience, 113, 881-890.

Buhusi, C. V., \& Schmajuk, N. A. (1996). Attention, configuration, and hippocampal function. Hippocampus, 6, 621-642.

Bunsey, M., \& Eichenbaum, H. (1993). Critical role for the parahippocampal region for paired-associate learning in rats. Behavioral Neuroscience, 107, 740-747.

Chan, K.-H., Morell, J. R., Jarrard, L. E., \& Davidson, T. L. (2001). Reconsideration of the role of the hippocampus in learned inhibition. Behavioural Brain Research, 119, 111-130.

Chen, G., \& Steinmetz, J. E. (1998). A general-purpose computer system for behavioral conditioning and neural recording experiments. Behavioral Research Methods, Instruments, \& Computers, 30, 384391.

Douglas, R. J. (1967). The hippocampus and behavior. Psychological Bulletin, 67, 416-442.

Douglas, R. J., \& Pribram, K. (1966). Learning and limbic lesions. Neuropsychologia, 4, 192-222.

Eichenbaum, H. (1999). The hippocampus: The shock of the new. Current Biology, 9, R482-R484.

FANSElow, M. S. (1998). Pavlovian conditioning, negative feedback, and blocking: Mechanisms that regulate association formation. $\mathrm{Neu}$ ron, 20, 625-627.

Gluck, M. A., Allen, M. T., Myers, C. E., \& Thompson, R. F. (2001). Cerebellar substrates for error-correction in motor conditioning. Neurobiology of Learning \& Memory, 76, 314-341.

GLuCK, M. A., \& Myers, C. E. (1993). Hippocampal mediation of stimulus representation: A computational theory. Hippocampus, 3, 491516.

Gormezano, I., Kehoe, E. J., \& Marshall, B. S. (1983). Twenty years of classical conditioning research with the rabbit. Progress in Psychobiology \& Physiological Psychology 10, 197-275.

Grossberg, W., \& Merrill, J. W. L. (1992). A neural network model of adaptively timed reinforcement learning and hippocampal dynamics. Cognitive Brain Research, 1, 3-38.

Hasselmo, M. E., \& Wyble, B. P. (1997). Free recall and recognition in a network model of the hippocampus: Simulating effects of scopolamine on human memory. Behavioural Brain Research. 89, 1-34.

Holland, P. C. (1997). Brain mechanisms for changes in processing of conditioned stimuli in Pavlovian conditioning: Implications for behavior theory. Animal Learning \& Behavior, 25, 373-399.

JARRARD, L. (1989). On the use of ibotenic acid to lesion selectively different components of the hippocampal formation. Journal of Neuroscience Methods, 29, 251-259.

Kamin, L. (1969). Predictability, surprise, attention and conditioning. In B. Campbell \& R. Church (Eds.), Punishment and aversive behavior (pp. 279-296). New York: Appleton-Century-Crofts.

KIM, J. J., KRUPA, D. J., \& Thompson, R. F. (1998). Inhibitory cerebelloolivary projections and blocking effect in classical conditioning. Science, 279, 570-573.

Kimble, D. P. (1968). Hippocampus and internal inhibition. Psychological Bulletin, 70, 285-295.

KINKAIDE, P. S. (1974). Stimulus selection in eyelid conditioning in the rabbit (Oryctolagus cuniculus). Journal of Comparative \& Physiological Psychology, 86, 1132-1140.

MackINTOSH, N. J. (1973). Stimulus selection: Learning to ignore stimuli that predict no change in reinforcement. In R. Hinde \& J. StevensonHinde (Eds.), Constraints on learning: Limitations and predispositions (pp. 75-96). New York: Academic Press.

Mackintosh, N. J., \& Turner, C. (1971). Blocking as a function of novelty of CS and predictability of UCS. Quarterly Journal of Experimental Psychology, 23, 359-366.

Marchant, H. G., III, \& Moore, J. W. (1973). Blocking of the rabbit's conditioned nictitating membrane response in Kamin's two-stage paradigm. Journal of Experimental Psychology, 101, 153-158.

Menon, V., White, C. D., Eliez, S., Glover, G. H., \& Reiss, A. L. 
(2000). Analysis of a distributed neural system involved in spatial information, novelty, and memory processing. Human Brain Mapping, 11, 117-129.

Miller, R. R. \& Matzel, R. D. (1988). The comparator hypothesis: A response rule for the expression of associations. In G. H. Bower (Ed.), Psychology of learning and motivation (pp. 51-92). New York: Academic Press.

Myers, C. E., Gluck, M. A., \& Granger, R. (1995). Dissociation of hippocampal and entorhinal function in associative learning: A computational approach. Psychobiology, 23, 116-138.

Pribram, K. H. (1986). Preface. In R. L. Isaacson \& K. H. Pribram (Eds.), The hippocampus (Vol. 4, pp. vii-xiii). New York: Plenum.

REsCorla, R, \& WAGNER, A. (1972). A theory of Pavlovian conditioning: Variations in the effectiveness of reinforcement and non-reinforcement. In A. H. Black \& W. F. Prokasy (Eds.), Classical conditioning II: Current research and theory (pp.64-99). New York: Appleton-CenturyCrofts.

Schmajuk, N. A., \&. Buhusi, C.V. (1997). Stimulus configuration, occasion setting, and the hippocampus. Behavioral Neuroscience, 111, 235-258.

Schmaltz, L. W., \& Theios, J. (1972). Acquisition and extinction of a classically conditioned response in hippocampectomized rabbits (Oryctolagus cuniculus). Journal of Comparative \& Physiological Psychology, 79, 328-333.

Shohamy, D., Allen, M. T., \& Gluck, M. A. (2000). Dissociating entorhinal and hippocampal function in latent inhibition. Behavioral Neuroscience, 114, 867-874.
Solomon, P. R. (1977). Role of the hippocampus in blocking and conditioned inhibition of the rabbit's nictitating membrane response. Journal of Comparative \& Physiological Psychology, 91, 407-417.

Solomon, P. R. (1980). A time and place for everything? Temporal processing views of hippocampal function with special reference to attention. Physiological Psychology, 8, 254-261.

Solomon, P. R., \& Moore, J.W. (1975). Latent inhibition and stimulus generalization of the classically conditioned nictitating membrane response in rabbits (Oryctolagus cuniculus) following hippocampal ablation. Journal of Comparative \& Physiological Psychology, 89, 11921203.

SQuire, L. R. (1992). Memory and the hippocampus: A synthesis from findings with rats, monkeys, and humans. Psychological Review, 99, 195-231.

Thompson, L. T., Moyer, J. R., Akase, E. \& Disterhoft, J. F. (1994). A system for quantitative analysis of associative learning: Pt. 1. Hardware interfaces with cross-species applications. Journal of Neuroscience Methods, 54, 109-117.

Thompson, R. F. (1986). The neurobiology of learning and memory. Science, 233, 941-947.

Vinogradova, O. S. (2001). Hippocampus as comparator: Role of the two input two output systems of the hippocampus in selection and registration of information. Hippocampus, 11, 578-598.

(Manuscript received January 16, 2002; revision accepted for publication July 16, 2002.) 